\title{
The influence of the nature of sound reflection from enclosures on the reverberation processes in the rooms
}

\author{
Aleksander Golovko ${ }^{1, *}$, Vladimir Ledenev ${ }^{2}$, and Aleksander Antonov ${ }^{2}$ \\ ${ }^{1}$ Far Eastern State Transport University, st. Serysheva 47, Khabarovsk, 680021, Russia \\ ${ }^{2}$ Tambov State Technical University, st. Sovetskaya 106, Tambov, 392000, Russia
}

\begin{abstract}
The article considers the questions of assessment of reverberation processes in rooms with various character of a sound reflection from the enclosures. An efficient technique for calculating of the reverberation sound energy in the rooms with mirror-diffuse reflection of sound is offered. The technique is based on the representation of mirror reflected sound rays in the form of separate groups with parameters corresponding oblique, axial and tangential rays. The article includes the comparison of the calculation of reverberation time results on the basis of the division of all the rays into groups with the results of direct reverberation modeling by tracing rays. A good convergence of the results was obtained.
\end{abstract}

\section{Introduction}

After the source is turned off, the sound field is attenuated, which means the reverberation process can be observed. The rate of sound attenuation or reverberation time represents an important acoustic characteristic of the room. It determines the main indicators of acoustic qualities of auditoriums, such as audibility and intelligibility of speech, as well as the quality of perception of music and other acoustic signals. In the practice of noise control in industrial and civil buildings, the reverberation processes are applied to obtain the acoustic characteristics of the rooms and, first of all, the sound absorption coefficients of enclosures. Reverberation processes are a special case of a more general problem of calculating and analyzing non-constant sound fields in rooms of noise sources with a time acoustic power variable. In this connection, the refinement of the method for calculating and analyzing the reverberation processes keeps the great practical importance, since it makes possible to improve the accuracy of obtaining sound-absorbing characteristics of enclosures and to improve the assessment of the acoustic parameters of auditoriums.

Calculation of the reverberation time of diffuse sound fields is carried out using the Sabine or Eyring formulas [1,2]. The conditions for the applicability of these formulas depend on the availability of a sufficient number of modes arising in the considered frequency range [3], and also are determined by the degree of fulfillment of the diffusivity conditions. In this case, the rooms should be commensurate with approximately the same sound-

*Corresponding authors: golovko@ festu.khv.ru 
absorbing properties of the enclosures. The degree of diffusion increases with the presence of sound scatterers, as well as in rooms of irregular geometric shapes $[4,5]$.

In the case of mirror reflection of sound from enclosures and the presence of large parallel reflecting surfaces, groups of slowly damped sound rays are formed in the rooms and, consequently, the reverberation time considerably exceeds the values obtained by the Sabine formula [6]. For such conditions, an expression was proposed in [7], which takes into account the different attenuation rates of groups of sound waves or modes. However, the proposed analytical expressions do not allow considering the influence of numerous factors on the magnitude of the reverberation time [8].

At present, computer methods, developed on the basis of ray tracing principles, are widely used for simulation of reverberation processes $[9,10,11]$. The methods basically realize the ideal mirror model of the sound reflection from enclosures, which does not always correspond to the real processes of sound reflection in the rooms. The article considers the principles of the assessment of reverberation processes in rooms at realization of the combined diffusive-mirror model of sound reflection from protections, that best corresponds to real conditions of sound reflection in rooms.

Reflection of sound from enclosures is carried out by complex spatial dependencies, determined by the shape of the surface, the structure of the material, the angle of incidence of sound and other parameters [12]. There is a limited class of rooms where reverberation processes are described with sufficient accuracy by ideal models of mirror (Fig. 1, a) or diffuse (Fig. 1, b) reflection of sound from enclosures. The accuracy of the calculation of the reverberation time depends on the degree of correspondence between the real and ideal models of sound reflection. In reality the sound reflection occurs through complex schemes, when some of the energy is reflected in a mirror image, while the other part scatters diffusely (Fig. 1, c). For this reason, in order to calculate the reflected sound fields of rooms we should apply the combined mirror-diffuse reflection models with a certain ratio between the scattered and mirror components of the reflected energy.
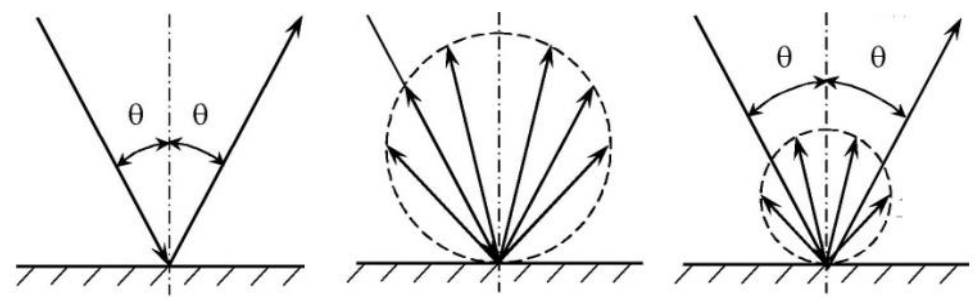

Fig. 1. Schemes of sound reflection from enclosures.

The combined mirror-diffuse nature of the reflection of sound from enclosures leads to the formation of two groups of sound rays in the room, which are the result of mirror and diffuse reflection. These ray groups have different formation conditions, conform different laws of propagation, accumulation and damping. The mirror-reflected rays are described by methods of geometric acoustics of rooms, diffusely reflected follow the laws of statistical theory.

In the case of mirror reflection of sound in rooms of a rectangular parallelepiped shape, stable groups of sound waves are formed, which according to [13] can be defined as axial, tangential and oblique with corresponding values of average values of mean free paths:

- for axial

$$
\bar{l}_{x}=l_{x} ; \bar{l}_{y}=l_{y} ; \bar{l}_{z}=l_{z}
$$

- for tangential 


$$
\bar{l}_{x y}=\frac{\pi l_{x} l_{y}}{2\left(l_{x}+l_{y}\right)} ; \bar{l}_{x z}=\frac{\pi l_{x} l_{z}}{2\left(l_{x}+l_{z}\right)} ; \bar{l}_{y z}=\frac{\pi l_{y} l_{z}}{2\left(l_{y}+l_{z}\right)} ;
$$

- for oblique

$$
\bar{l}_{x y z}=4 V / S=2 l_{x} l_{y} l_{z} /\left(l_{x} l_{y}+l_{x} l_{z}+l_{y} l_{z}\right),
$$

where $l_{x}, l_{y}, l_{z}-$ length, width and height of the room.

The initial energy distribution in percent $k_{j}$ of total energy between groups of rays is determined mainly by the shape of the room. As an example, in Table 1 the given values of $k_{j}$ are calculated for use in mirror reflection of sound from enclosures.

Table 1. Energy distribution by axial, tangential and oblique groups of rays as a percentage of total

\begin{tabular}{|c|c|c|c|c|c|c|}
\hline \multicolumn{7}{|c|}{ Room $3 \times 3 \times 3 \mathrm{~m}$} \\
\hline \multicolumn{3}{|c|}{ Axial $-10.4 \%$} & \multicolumn{3}{|c|}{ Tangential-38.8\% } & Oblique $-50.8 \%$ \\
\hline $\begin{array}{l}\text { For X } \\
\bar{l}_{x}=3 \mathrm{~m}\end{array}$ & $\begin{array}{c}\text { For Y } \\
\bar{l}_{y}=3 \mathrm{~m}\end{array}$ & $\begin{array}{c}\text { For Z } \\
\bar{l}_{z}=3 \mathrm{~m}\end{array}$ & $\begin{array}{c}\text { For XY } \\
\bar{l}_{x y}=2,36 \mathrm{~m}\end{array}$ & $\begin{array}{c}\text { For XZ } \\
\bar{l}_{x z}=2,36 \mathrm{~m}\end{array}$ & $\begin{array}{c}\text { For YZ } \\
\bar{l}_{y z}=2,36 \mathrm{~m}\end{array}$ & $\begin{array}{c}\text { For XYZ } \\
\bar{l}_{x y z}=2 \mathrm{~m}\end{array}$ \\
\hline $3.5 \%$ & $3.4 \%$ & $3.5 \%$ & $12.9 \%$ & $12.9 \%$ & $13 \%$ & $50.8 \%$ \\
\hline \multicolumn{7}{|c|}{ Room 9x3x3 m } \\
\hline \multicolumn{3}{|c|}{ Axial-17.7\% } & \multicolumn{3}{|c|}{ Tangential- $45.8 \%$} & Oblique $-36.5 \%$ \\
\hline $\begin{array}{c}\text { For X } \\
\bar{l}_{x}=9 \mathrm{~m}\end{array}$ & $\begin{array}{c}\text { For Y } \\
\bar{l}_{y}=3 \mathrm{~m}\end{array}$ & $\begin{array}{c}\text { For Z } \\
\bar{l}_{z}=3 \mathrm{~m}\end{array}$ & $\begin{array}{c}\text { For XY } \\
\bar{l}_{x y}=3.53 \mathrm{~m}\end{array}$ & $\begin{array}{c}\text { For XZ } \\
\bar{l}_{x z}=3.53 \mathrm{~m}\end{array}$ & $\begin{array}{c}\text { For YZ } \\
\bar{l}_{y z}=2.36 \mathrm{~m}\end{array}$ & $\begin{array}{c}\text { For XYZ } \\
\bar{l}_{x y z}=2.57 \mathrm{~m}\end{array}$ \\
\hline $0.4 \%$ & $8.7 \%$ & $8.6 \%$ & $5.8 \%$ & $5.8 \%$ & $34.2 \%$ & $36.5 \%$ \\
\hline \multicolumn{7}{|c|}{ Room 9x9x3 m } \\
\hline \multicolumn{3}{|c|}{ Axial-23.9\% } & \multicolumn{3}{|c|}{ Tangential $-37.7 \%$} & Oblique $-38.4 \%$ \\
\hline $\begin{array}{l}\text { For } \mathrm{X} \\
\bar{l}_{x}=9 \mathrm{~m}\end{array}$ & $\begin{array}{c}\text { For Y } \\
\bar{l}_{y}=9 \mathrm{~m}\end{array}$ & $\begin{array}{c}\text { For Z } \\
\bar{l}_{z}=3 \mathrm{~m}\end{array}$ & $\begin{array}{c}\text { For XY } \\
\bar{l}_{x y}=7.07 \mathrm{~m}\end{array}$ & $\begin{array}{c}\text { For XZ } \\
\bar{l}_{x z}=3.53 \mathrm{~m}\end{array}$ & $\begin{array}{c}\text { For YZ } \\
\bar{l}_{y z}=3.53 \mathrm{~m}\end{array}$ & $\begin{array}{c}\text { For XYZ } \\
\bar{l}_{x y z}=3.6 \mathrm{~m}\end{array}$ \\
\hline $1.1 \%$ & $1.1 \%$ & $21.7 \%$ & $4.4 \%$ & $16.7 \%$ & $16.6 \%$ & $38.4 \%$ \\
\hline
\end{tabular}
energy.

The accumulation of energy of sound rays with prolonged action of the sound source is affected by the reflecting properties of the enclosing surfaces encountered in the way of the rays.

The formula for the average coefficient of sound absorption of surfaces from which the axial rays, propagating along $\mathrm{X}$ axis are reflected, has the form

$$
\bar{\alpha}_{x}=1-\sqrt{\left(1-\alpha_{x}^{\prime}\right)\left(1-\alpha_{x}^{\prime \prime}\right)}
$$

where $\alpha_{x}, \alpha{ }_{x}$ - coefficients of sound absorption of the ends of the room.

The average coefficient of sound absorption of the enclosures in the way of tangential ray propagating in the horizontal plane, taking into account the probability of interaction of the rays with the surfaces of different areas, is presented by the formula

$$
\bar{\alpha}_{x y}=1-\left(1-\alpha_{x}^{\prime}\right)^{l_{y} / P_{x y}}\left(1-\alpha_{y}^{\prime \prime}\right)^{l_{x} / P_{x y}}\left(1-\alpha_{x}^{\prime \prime}\right)^{l_{y} / P_{x y}}\left(1-\alpha_{y}^{\prime}\right)^{l_{x} / P_{x y}}
$$


where $P_{x y}=2\left(l_{x}+l_{y}\right)-$ cross-section perimeter of the room;

$\alpha_{y}, \alpha_{y}{ }_{y}$ - coefficients of sound absorption of the side surfaces of the room.

Similar expressions are written for average coefficients of sound absorption of surfaces along the way of other axial and tangential rays. rays is

The general formula for recording the average sound absorption coefficient for oblique

$$
\bar{\alpha}_{x y z}=1-\prod_{j=1}^{6}\left(1-\alpha_{j}\right)^{S_{j} / S},
$$

where $\alpha_{\mathrm{j}}$ - coefficient of sound absorption of the surface area $S_{j}$;

$S$ - total area of all enclosures.

Formulas (1) - (6) allow us to calculate the parameters of the sound field in a rectangular room with a mirror image of the sound from the enclosures:

- value of the average density of reflected energy produced by a group of rays

$$
\varepsilon_{o j}^{3}=\sum_{i=1}^{\infty} \frac{W k_{j} \bar{l}_{j}\left(1-\bar{\alpha}_{j}\right)^{i}}{V c}=\frac{W k_{j} \bar{l}_{j}\left(1-\bar{\alpha}_{j}\right)}{\bar{\alpha}_{j} V c}
$$

- formulas for attenuation of sound energy after switching off the sound source of a constant acoustic power

$$
\varepsilon_{t}^{3}=\sum_{j=1}^{N} \varepsilon_{o j}^{3}\left(1-\bar{\alpha}_{j}\right)^{t c / \bar{l}_{j}}
$$

In formulas (7) and (8) we use the symbols: $W$ is the acoustic power of the sound source, $\mathrm{W} ; c$ is the speed of sound, $\mathrm{m} / \mathrm{s} ; t$ - time after disconnection of the sound source, $\mathrm{s} ; k_{j}$ is the part of the energy of the sound source that belongs to the group of rays with an average mean free path $l_{j}$ and the average absorption coefficient $\alpha_{j}$.

Figure 2 shows the results of comparing computer simulation of reverberation processes by ray tracing and calculating sound attenuation by ray groups based on formula (8) for rooms of various proportions with a muffled ceiling. As expected, the attenuation of the energy is much slower than the attenuation determined by Eyring equation for the diffuse reflection of sound. In this case, there is a good agreement between computer simulation and calculation of reverberation in formula (8), taking into account the decomposition of the energy of the sound field into groups of rays with the same characteristics.

In the case of diffuse reflection of sound, the front of sound waves is fragmented and, accordingly, intense mixing of the rays due to the complex shape of the rooms, the presence of sound-scattering elements, equipment, and other factors [14]. It is noted in [13] that in such cases all rays should be assumed to be oblique with average mean free path, determined by the formula (3). The average coefficient of sound absorption of rooms in this case is calculated according to the diffuse theory by formula

$$
\bar{\alpha}=\frac{\sum_{j=1}^{6} \alpha_{j} S_{j}}{\sum_{j=1}^{6} S_{j}}
$$

The graphs of Figure 2 clearly show the differences in the processes of attenuation of sound energy in the mirror and diffuse nature of the reflection of sound from enclosures 

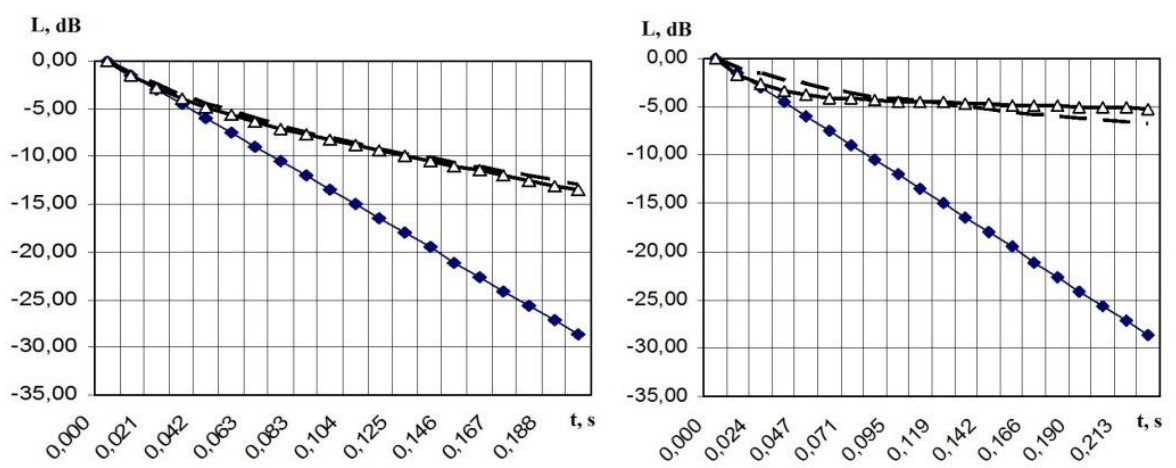

Fig. 2. Reverberation graphs in long and flat rooms with proportions. (a) 12:1:1, b) 12:12:1): calculation at diffuse reflection of sound; $-\Delta-$ - calculation at mirror reflection with division of rays into groups (axial, tangential, oblique); - - --- ray tracing method.

Rays that are reflected from the enclosures mirror and diffusely participate simultaneously at combined mirror-diffuse reflection of sound in the formation of the sound field.

Figure 3 shows an example of a change in the structure of the density of the reflected energy of individual pulse after several acts of reflection $\mathrm{N}_{\text {отр }}$ and partial scattering during a mirror-diffuse reflection of sound from enclosures. The graphs of Fig. 3 show that at the initial instant of time after radiation the fraction of the mirror component of the pulse is sufficiently high. However, after several acts of reflection, its part is sharply reduced. The proportion of scattered energy increases as a result of each act of reflection and partial scattering of mirror energy. After several reflections the scattered reflected energy exceeds the mirror energy, and at the final stage of the reverberation processes the diffusely reflected energy becomes dominant.

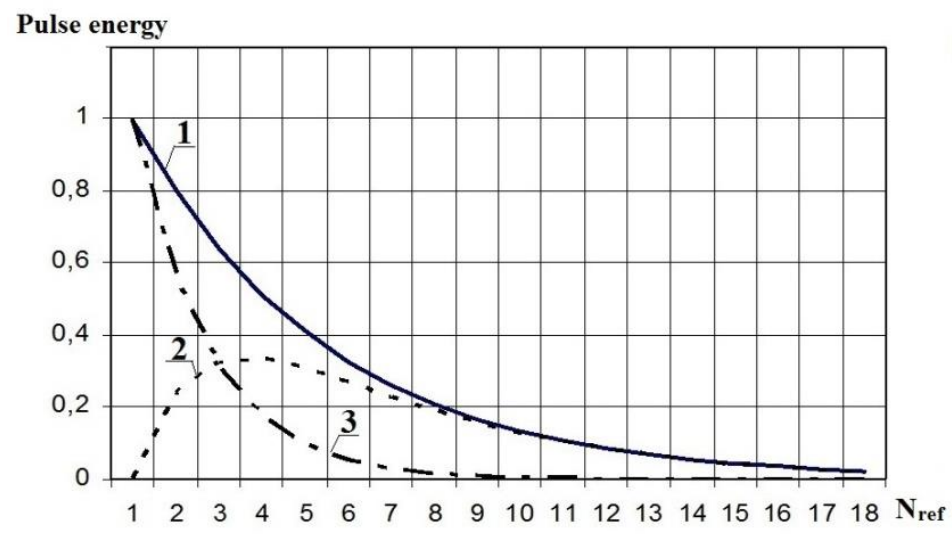

Fig. 3. The structure of change of individual pulse in the mirror-diffuse nature of the reflection of sound in fractions of the original energy: 1 - total reflected energy; 2 - diffuse energy; 3 - mirrorreflected energy.

Figure 4 shows the process of increasing the density of reflected energy in a room after switching on a constant noise source as a result of summing the contribution of a large number of radiated energy pulses. It can be seen that with a steady sound field, the diffusely reflected sound energy exceeds the specularly reflected energy. 


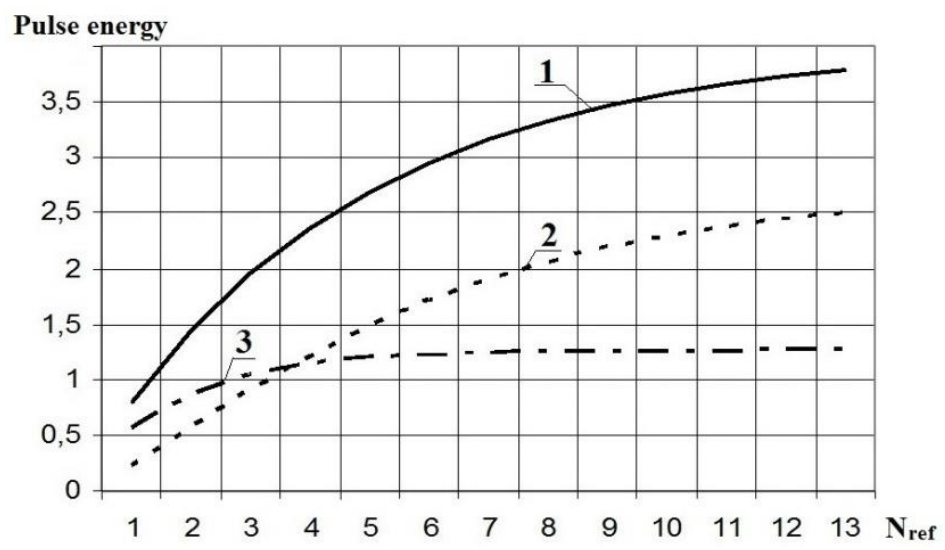

Fig. 4. The graph of the increase of total, mirror and scattered components of the reflected field when a long-acting noise source is switched on in proportions of the energy of individual pulses: 1 - total reflected energy; 2 - diffuse energy; 3 - mirror-reflected energy.

The average density of reflected sound energy generated by the mirror component is calculated by the formula

$$
\varepsilon_{o j}^{3}=\sum_{i=1}^{\infty} \frac{W k_{j} \bar{l}_{j}\left(1-\bar{\alpha}_{j}\right)^{i}(1-\beta)^{i}}{V c}=\frac{W k_{j} \bar{l}_{j}\left(1-\bar{\alpha}_{j}\right)(1-\beta)}{\left(\bar{\alpha}_{j}+\beta-\bar{\alpha}_{j} \beta\right) V c}
$$

where $\beta$ - coefficient that shows part of the energy reflected diffusely in the mirror-diffuse nature of the reflection of sound from enclosures [15].

Diffusely reflected from the enclosures, the energy of the sound rays equals

$$
\varepsilon_{o j}^{p}=\frac{W k_{j} \bar{l}\left(1-\bar{\alpha}_{j}\right) \beta}{\bar{\alpha}\left(\bar{\alpha}_{j}+\beta-\bar{\alpha}_{j} \beta\right) V c}
$$

The total density of reflected sound energy, which is created by mirror and diffuse reflections, equals

$$
\varepsilon_{o j}=\varepsilon_{o j}^{3}+\varepsilon_{o j}^{p}=\frac{W k_{j} \bar{l}_{j}\left(1-\bar{\alpha}_{j}\right)(1-\beta)}{\left(\bar{\alpha}_{j}+\beta-\bar{\alpha}_{j} \beta\right) V c}\left(1+\frac{\bar{l} \beta}{\bar{\alpha} \bar{l}_{j}(1-\beta)}\right)
$$

The formulas for the description of the attenuation of mirror and scattered sound energy after switching off the sound source of constant acoustic power

$$
\begin{gathered}
\varepsilon_{t}^{3}=\sum_{j=1}^{N} \varepsilon_{o j}^{3}\left(1-\bar{\alpha}_{j}\right)^{t c / \bar{l}_{j}}(1-\beta)^{t c / \bar{l}_{j}} \\
\varepsilon_{t}^{p}=\sum_{j=1}^{N}\left(\varepsilon_{o j}^{p}(1-\bar{\alpha})^{t c / \bar{l}_{c p}}+\sum_{i=1}^{t c / \bar{l}_{j} t c / \bar{l}_{\mathrm{cp}}} \sum_{m=i}^{3} \varepsilon_{o j}^{3}\left(1-\bar{\alpha}_{j}\right)^{i} \beta(1-\bar{\alpha})^{m}\right) .
\end{gathered}
$$

The formula (14) takes into account the transition of a part of the mirror component of the field to the scattered one at each reflection act.

The formulas (13) and (14) make it possible to estimate the attenuation of sound energy in the mirror-diffuse nature of the reflection of sound, taking into account the space-planning and acoustic parameters of the rooms. 
Below, as an example, the results of calculating the attenuation of sound energy in a room with sizes 10x6x3 m are given. The coefficient of sound absorption of the ceiling of the room $\alpha_{\text {пा }}=0.10$, coefficient of diffuse reflection of sound energy $\beta=0.10$.

The parameters of the groups of rays mirror reflected are given in Table 2.

Table 2. Acoustic parameters of mirror reflected rays.

\begin{tabular}{|l|c|c|}
\hline \multicolumn{1}{|c|}{ Name of group of rays } & $\begin{array}{c}\text { Average mean free path, } \\
\mathrm{m}\end{array}$ & $\begin{array}{c}\text { Average coefficient of } \\
\text { sound absorption }\end{array}$ \\
\hline Axials along the axis $\mathrm{X}$ & 10 & 0.1 \\
\hline Axials along the axis $\mathrm{Y}$ & 6 & 0.1 \\
\hline Axials along the axis $\mathrm{Z}$ & 3 & 0.48 \\
\hline Tangents in the plane XY & 5.89 & 0,1 \\
\hline Tangents in the plane XZ & 3.62 & 0,410 \\
\hline Tangents in the plane YZ & 3.14 & 0,376 \\
\hline Oblique & 3.33 & 0,337 \\
\hline
\end{tabular}

Figure 5 shows the energy decrease rates of various groups of rays when they are mirror reflected, taking into account the average sound absorption coefficient of enclosures in the path of these rays and the partial scattering of reflected sound by enclosures

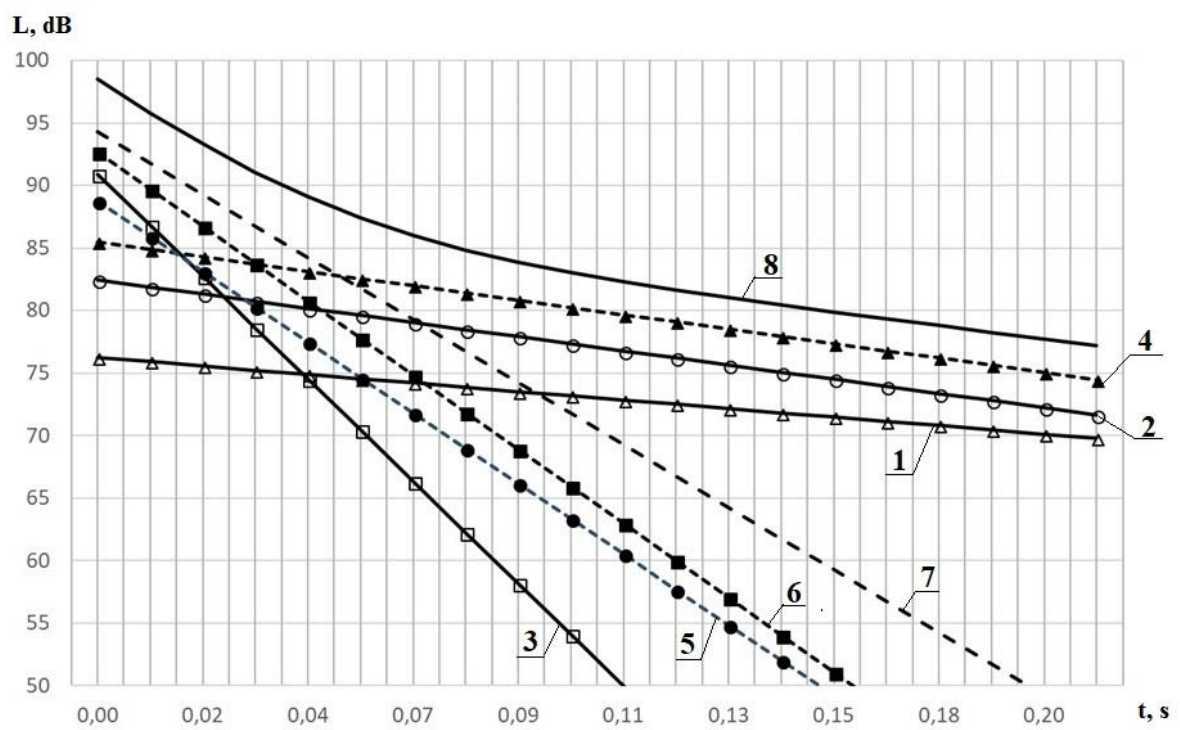

Fig. 5. Attenuation of various groups of mirror reflected sound rays: 1 - axial along the $X$ axis, 2 axial along the $\mathrm{Y}$ axis, 3 - axial along the $\mathrm{Z}$ axis,4- tangents in the $\mathrm{XY}$ plane, 5 - tangents in the $\mathrm{XZ}$ plane, 6 - tangents in the YZ plane, 7 - oblique; 8 - total attenuation.

Figure 6 shows the decrease of mirror and diffusely reflected sound energy. For the diffusely reflected energy two graphs are given. Line 2 shows the attenuation of the diffusely reflected energy accumulated in the room with a long-acting sound source, that is, the damping of the diffuse energy of the stationary sound field. This energy was formed due to the scattering of sound at each act of reflection. The damping of this part of the energy corresponds to Eyring equation. After the sound is turned off, a part of the sound energy, generated as a result of the partial dissipation of the mirror energy, will be added to the diffusely reflected sound at each act of reflection. The total amount of scattered energy, taking into account the continuous flow of the sound energy of the specularly reflected rays 
into the diffuse energy, is shown in Fig. 6 in the form of line 3. The attenuation of this energy appears to be slower than it occurs according to Eyring equation.

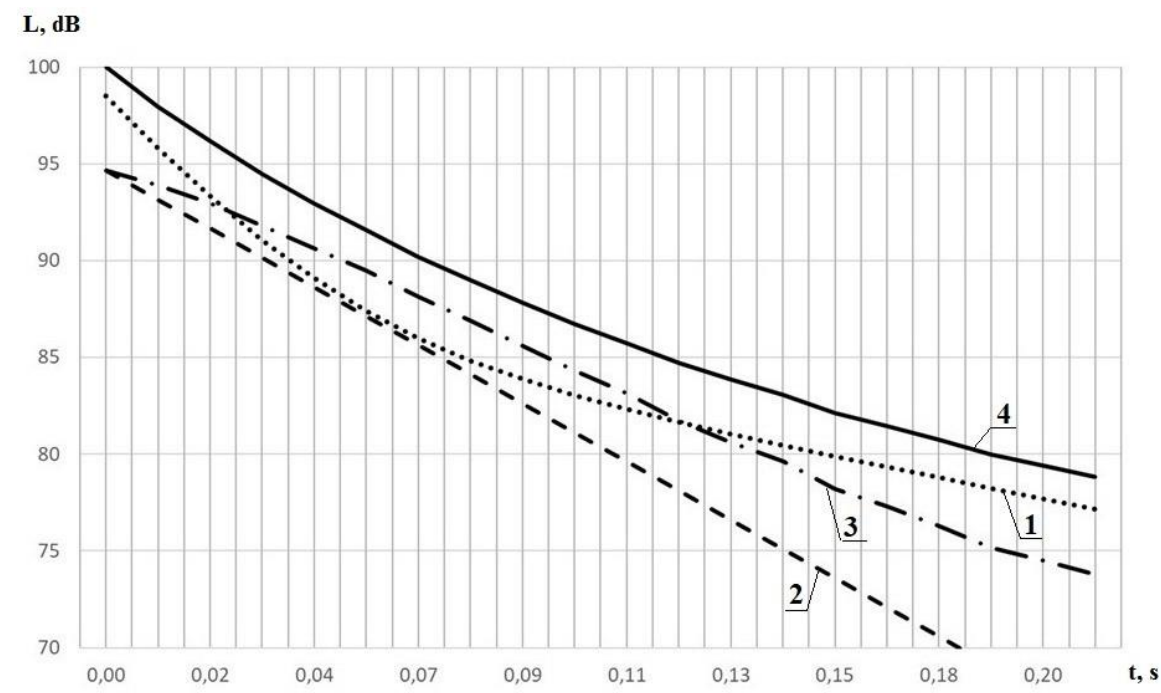

Fig. 6. Attenuation of the total, diffuse and mirror reflected sound energy with a combined scheme of sound reflection from the enclosures of the room: 1 - mirror reflected energy;

2-diffuse energy of stationary sound field; 3 - total diffuse energy with the partial scattering of the specularly reflected energy in the process of reverberation; 4 - total reflected energy.

The analysis of the graphs in Fig. 6 shows that the nature of the attenuation of the total reflected energy in the conditions of real rooms with mirror-diffuse reflection of sound from enclosures depends significantly on the damping of energy transferred by different groups of specularly reflected sound rays. The rate of attenuation in each group depends on the mean free path of the group rays and the average sound absorption coefficient for it.

At the initial stage the rate of attenuation of the total energy is determined mainly by the diffuse component of energy and the energy of the group of oblique mirror-reflected rays. This section of the attenuation curve should be used when finding the average sound absorption coefficient of the room according to the results of the experimentally determined reverberation time.

The subsequent sections of the decline curve of the total energy are mainly determined by slower damping of the groups of tangential and axial rays, as well as by a slower decline of the diffusely reflected energy.

At the last stage, the decline of the total energy is determined by the energy of the axial rays. The attenuation of these rays determines in many respects the residual rustle of the room.

Since the different groups of mirror rays affect the reverberation process indoors in different ways, the attenuation of sound energy is largely determined by the volume-planning parameters and the sound absorption coefficients of the room enclosures. Concrete increase in sound absorption in certain areas of enclosures makes it possible to change the nature of the attenuation of sound energy at different times of the reverberation process. For example, it is necessary to perform sound-absorbing lining on the end walls to reduce the noise in long rooms. This will speed up the attenuation of a group of long axial rays and thereby reduce the reverberation time.

Overall, the results of the work allow us to draw the following conclusions.

Comparing the results of calculating the reverberation in rooms of different proportions, the mirror and diffuse reflection of sound from the enclosures show a significant discrepancy. 
It is established that in real rooms the reflection of sound from enclosures occurs according to complex dependencies, which are best modeled by a mirror-diffuse reflection model. Direct modeling of reverberation processes by ray tracing with a mirror-diffuse reflection model leads to significant computational costs.

An effective technique for calculating reverberation in rooms with mirror-diffuse reflection of sound from enclosures is developed, based on the division of all mirror-reflected sound rays into groups with similar parameters along the mean free paths, the average sound absorption coefficients of enclosures, from which the rays are reflected and, accordingly, with equal degree attenuation. The minimum number of ray groups corresponds to axial, tangential and oblique rays.

The results of calculating the reverberation time are compared on the basis of dividing all the rays into groups with the results of direct reverberation modeling by ray tracing. There is good agreement of the results. The proposed technique for determining the reverberation time is suitable for solving practical problems.

\section{References}

1. W.C. Sabine, Collected papers an acoustics (Dower publications, New York, 1964)

2. C.F. Eyring, Journal of the Acoustical Society 1, 217-241 (1930)

3. M.R. Schroeder, Measurement of Sound diffusion in reverberation Chambers 31-NII, 1407-1414 (1959)

4. H. Kuttruff, Zur Abhänqigkeit der Raumnachhalls von deu Wand diffusitat und von Raumform, Acustica 45- N4, 246-255 (1980)

5. I. Cremer, Die wissenschaftlichen Grundlagen der Raumakustik (Hirzel Ferlag, Stuttgart, 1976)

6. H. Kuttruff, Eigenschaften und Answertung von nachhallkurven, Acustica 8, 273-280 (1958)

7. D. Fitzroy, Reverberation formula which scems to be more accurate with non-uniform distribution, JASA 31, 893-897 (1959)

8. M.V. Sergeev, Acoustic journal 15-4, 591-598 (1979)

9. V.A. Yezersky, Scientific Journal of Construction and Architecture 2, 102-109 (2008)

10. Ya.S. Vakhitov, D.A. Davydov, Fundamental researches 4, 32-36 (2015)

11. I. Bork, Acta Acustica 91, 753-763 (2005)

12. A.I. Antonov, Privolzhsky scientific journal 2(42), 16-23 (2017)

13. F. Morz, Oscillation and Sound (Gostehteorizdat, Leningrad, 1949)

14. A.I. Antonov, Privolzhsky scientific journal 3(35), 89-96 (2015)

15. B.I. Giyasov, Engineering and construction magazine 1(77), 13-22 (2018) 